\title{
Jeder zehnte Arzt steigt aus
}

\author{
Esther Kraft ${ }^{\mathrm{a}}$, Lisa Loretan ${ }^{\mathrm{b}}$, Nico van der Heiden ${ }^{\mathrm{c}}$ \\ ${ }^{a}$ Leiterin Abteilung Daten, Demographie und Qualität DDQ, FMH; ${ }^{b}$ Projektassistentin Politik \& Kommunikation, VSAO; ${ }^{c}$ Stv. Geschäftsführer / Leiter Politik \\ und Kommunikation, VSAO
}

Wie viele Ärztinnen und Ärzte geben pro Jahr die kurative Tätigkeit am Patienten auf und weshalb? Diese Fragen haben der VSAO und die FMH in einer repräsentativen Studie untersuchen lassen. Dabei zeigt sich, dass bis zum Pensionsalter rund 10\% der Ärzte pro Abschlussjahrgang vorzeitig aus der Behandlung von Patienten aussteigen. Die Gründe überraschen nicht: Am häufigsten führen Arbeitspensum, Arbeitszeiten oder schlechte Vereinbarkeit von Familie und Beruf zum Ausstieg.

\section{Einleitung}

Die Frage nach der Anzahl Ärztinnen und Ärzte, welche nach Abschluss ihrer Ausbildung nicht mehr kurativ tätig sind, war in jüngster Vergangenheit immer wieder Thema in den Medien. Einigkeit besteht in der Frage, dass der Ausstieg aus dem Arztberuf am Patienten wenn immer möglich verhindert werden sollte. Gerade im Hinblick auf den zunehmenden Ärztemangel ist es zu vermeiden, dass Ärzte ihre kurative Tätigkeit am Patienten aufgeben.

Leider fehlen jedoch in dieser politisch relevanten Diskussion erhärtete Zahlen zur effektiven Zahl der Ärzte, welche nicht mehr kurativ tätig sind, zu den Gründen für die berufliche Neuorientierung sowie zum Verbleib der Berufsaussteigerinnen und -aussteiger. Deshalb haben der Verband der Assistenz- und Oberärztinnen und -ärzte (VSAO) und die Verbindung der Schweizer Ärztinnen und Ärzte (FMH) sich entschieden, wissenschaftlich fundierte Aussagen bereitzustellen. Das Büro Vatter und gfs.bern erhielten den Auftrag, die Thematik des Ausstiegs der Ärztinnen und Ärzte aus der kurativen Tätigkeit zu untersuchen.

\section{Schätzung der nicht kurativ tätigen Ärzte}

Die Analyse schliesst alle Ärztinnen und Ärzte ein, welche zwischen 1980 und 2009 in der Schweiz ihr Ärztediplom erworben haben (N=22356). Das Jahr 2009 wurde so gewählt, dass alle in die Analyse eingeschlossenen Ärzte zumindest die Weiterbildung abgeschlossen haben. Für die Schätzung des Anteils der Ärzte, welche die kurative Tätigkeit vor Erreichen des Pensionsalters aufgegeben haben, waren zwei Arbeitsschritte notwendig: die Plausibilisierung und die Projektion. Erstens wurde der Anteil für die Grundgesamtheit (Personen mit Hinweisen auf eine mögliche Aufgabe der kurativen Tätigkeit) basierend auf der Befragung eruiert und mittels multivariater statistischer Analyse plausibilisiert. Die Plausibilisierung erfolgt anhand der Gewichtung und der «Multilevel Regression and Poststratification (MRP)». In einem zweiten Schritt wurden die Eruierungen ergänzt durch eine Schätzung für die "Gegengruppe». Diese setzt sich aus Ärzten zusammen, bei denen keine erhöhte Wahrscheinlichkeit zur Aufgabe der kurativen Tätigkeit besteht.

Mit der Plausibilisierung und der Projektion konnten drei Szenarien für den Anteil der nicht mehr kurativen Ärztinnen und Ärzte modelliert werden:

Der Analyse zufolge ist unter den Frauen der Anteil der nicht mehr kurativ tätigen Ärzteschaft etwas höher als unter den Männern. Je nach Szenario liegt die Quote bei den Frauen 1,2 bis 1,6 Mal über derjenigen der Männer.

Für die grosse Mehrheit ist der Ausstieg aus der kurativen Tätigkeit am Patienten definitiv. Gemäss der Befragung hält es nur etwa jeder Zehnte für eher oder sehr

Tabelle 1: Anteil nicht mehr kurativ tätiger Ärzte nach Geschlecht und Sprachregion.

\begin{tabular}{lccccrrr}
\hline & Alle & Männer & Frauen & \multicolumn{2}{c}{$\begin{array}{l}\text { Deutsch- } \\
\text { schweiz }\end{array}$} & Westschweiz & Tessin \\
\hline Tiefes Szenario & $8,4 \%$ & $7,9 \%$ & $9,3 \%$ & $8,8 \%$ & $6,0 \%$ & $7,5 \%$ \\
\hline Mittleres Szenario & $10,7 \%$ & $8,9 \%$ & $12,7 \%$ & $10,3 \%$ & $9,7 \%$ & $9,4 \%$ \\
\hline Hohes Szenario & $12,9 \%$ & $9,9 \%$ & $16,1 \%$ & $11,8 \%$ & $13,5 \%$ & $11,2 \%$ & \\
\hline
\end{tabular}




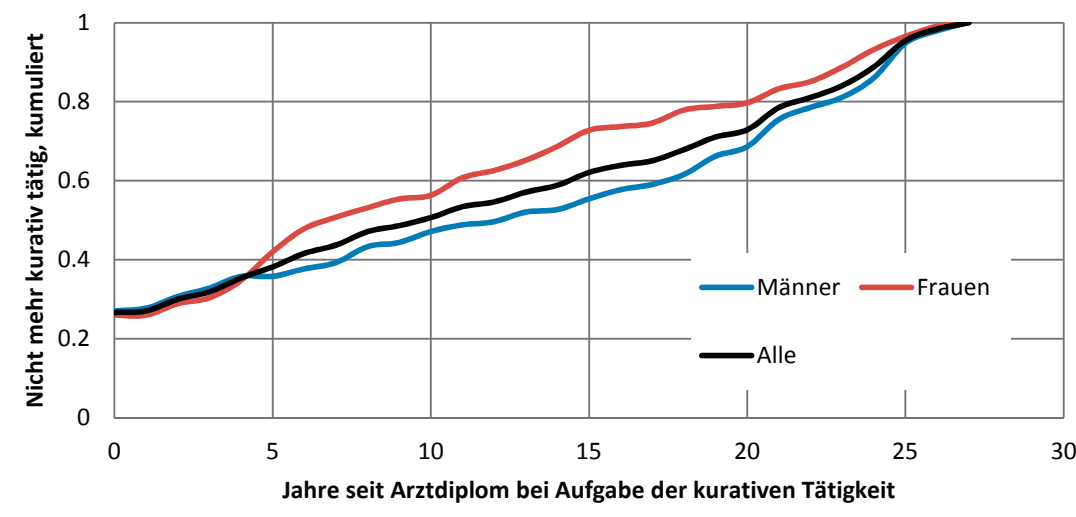

Quelle: gfs.bern, Laufbahnbefragung von Schweizer ÄrztInnen 2016. N = 195: Befragte mit Arztdiplom vor 1990, die nicht mehr kurativ tätig sind und deren Ausstieg spätestens 27 Jahre ab Arztdiplom erfolgte. Detailauswertung zu Frage 3 («Haben Sie nach dem Abschluss des Medizinstudiums eine ärztliche Weiterbildung angetreten?») und zu Frage 6 («Sind Sie aktuell kurativ am Patienten tätig? Es spielt keine Rolle, ob sie dabe Vollzeit oder Teilzeit arbeiten»).

Abbildung 1: Zeitpunkt der Aufgabe der kurativen Tätigkeit nach Geschlecht.

wahrscheinlich, wieder in den Arztberuf am Patienten zurückzukehren.

Aufgrund des mittleren Szenarios sind von den 22356 Ärztinnen und Ärzten, welche zwischen 1980 und 2009 ihr Ärztediplom in der Schweiz erworben haben, 2392 Personen nicht mehr kurativ tätig. Dies entspricht rund 80 Ärzten pro Abschlussjahrgang Humanmedizin.

\section{Entwicklung der ärztlichen Laufbahn}

Nachfolgend wird basierend auf der Umfrage detaillierter auf den Zeitpunkt eingegangen, zu welchem die Ärztinnen und Ärzte im Verlauf ihrer Karriere die kurative Tätigkeit aufgegeben haben. Weiter wird beschrieben, wie sich die Laufbahn der Ärzte entwickelt hat. Von der Grundgesamtheit $(\mathrm{N}=4023)$ konnten 3333 Ärzte postalisch oder per E-Mail kontaktiert werden. 1141 Interviews wurden realisiert, was einer Ausschöpfungsquote von 34,2 Prozent entspricht. Der Stichprobenfehler liegt bei \pm 3 Prozent.

Für die Gruppe der Ärzte, welche zwischen 1980 und 1989 ihr Arztdiplom erworben haben und nicht mehr kurativ tätig sind, ergibt sich folgendes Bild (Abb. 1): Nimmt man die beiden Geschlechter zusammen, so verläuft der Anstieg der Kurve ziemlich linear. Dies bedeutet, dass in jedem Jahr etwa derselbe Anteil an Ärzten hinzukommt, welche nicht mehr kurativ tätig sind. Etwas mehr als ein Viertel der Ausstiege erfolgt vor Aufnahme der Weiterbildung zum Facharzt. Die Kurven der Frauen und Männer nehmen zu Beginn der Laufbahn einen ähnlichen Verlauf. Nach rund vier Jahren, also während der Weiterbildung, beginnt der Anteil bei den Frauen beschleunigt anzusteigen. Erst ca. 25 Jahren nach dem Arztdiplom nähern sich die beiden Kurven wieder an.
Von den Ärzten, welche nicht kurativ tätig sind $(\mathrm{N}=180)$, haben ein Viertel angegeben, im Spital oder in anderen Institutionen der Gesundheitsversorgung tätig zu sein. 18 Prozent arbeiten in der Wissenschaft/ Forschung, 17 Prozent in der Lehre/Weiter- und Fortbildung, 17 Prozent in der Gesundheitsförderung/ Prävention, 13 Prozent in der öffentlichen Verwaltung und 13 Prozent in der Pharmaindustrie/Medizinaltechnik. Die restlichen Ärzte sind bei Versicherungen, Medien, Verbänden etc. tätig.

\section{Vier Typen von Ärzten ohne kurative Tätigkeit}

Berücksichtigt man einerseits den Zeitpunkt, zu welchem die Ärzte ihre kurative Tätigkeit aufgegeben haben, und andererseits ihre aktuelle Tätigkeit, so ergeben sich vier Typen:

- Typ 1: Frühe Berufswechselnde (46\% der nicht mehr kurativ tätigen Ärzte):

Kein Facharzttitel erworben und Qualifikation nützlich für die aktuelle Tätigkeit

- Typ 2: Frühe Berufsaussteigende (20\% der nicht mehr kurativ tätigen Ärzte):

Kein Facharzttitel und primär nicht berufstätig

- Typ 3: Späte Berufswechselnde (15\% der nicht mehr kurativ tätigen Ärzte):

Facharzttitel erworben und Qualifikation nützlich für die aktuelle Tätigkeit

- Typ 4: Späte Berufsaussteigende (19\% der nicht mehr kurativ tätigen Ärzte):

Facharzttitel erworben und primär nicht berufstätig. Es zeigt sich ein deutlicher und statistischer signifikanter Zusammenhang: Erfolgt die Aufgabe der kurativen Tätigkeit vor dem Erreichen des Facharzttitels, so geht die Mehrheit aktuell einem Beruf nach, in dem die ärztliche Qualifikation nützlich ist. Erfolgt hingegen die Aufgabe nach dem Facharzttitel, so hat für die aktuelle Tätigkeit die ärztliche Qualifikation häufiger keinen direkten Nutzen mehr. Nicht mehr kurativ tätigen Männern gelingt es besser als Frauen, ihre ärztliche Qualifikation auszuschöpfen: Sie arbeiten in Berufen, in denen diese nützlich oder sogar Voraussetzung ist, während Frauen etwas öfter nicht mehr berufstätig sind.

Von den ausgestiegenen Ärzten betrachtet es rund ein Zehntel (9\%) als sehr oder eher wahrscheinlich, wieder in die kurative Tätigkeit zurückzukehren. Bei den Frauen liegt der Anteil, der eine Rückkehr für mindestens «eher wahrscheinlich» hält, mit 14 Prozent höher als die 4 Prozent der Männer

Die noch kurativ tätigen Ärzte in Weiterbildung wurden nach der Wahrscheinlichkeit gefragt $(\mathrm{N}=204)$, dass sie die Weiterbildung mit dem Facharzttitel abschliessen. 


\begin{tabular}{|c|c|c|c|c|c|}
\hline & Alle & Typ 1 & Typ 2 & Typ 3 & Typ 4 \\
\hline Pensum und Arbeitszeiten & 34 & 43 & 34 & 37 & 11 \\
\hline \multirow{2}{*}{$\begin{array}{r}\text { Vereinbarkeit mit der Kinderbetreuung } \\
\text { Arbeitsinhalte }\end{array}$} & 22 & 27 & 32 & 16 & 9 \\
\hline & 21 & 27 & 11 & 34 & 5 \\
\hline Anforderungsniveau der Arbeit & 16 & 19 & 16 & 13 & 13 \\
\hline Gesundheitliche Gründe & 16 & 8 & 23 & 15 & 31 \\
\hline Neuorientierung, andere Interessen & 16 & 22 & 11 & 18 & 4 \\
\hline Ruhestand, Pensionierung & 15 & 1 & 14 & 4 & 59 \\
\hline $\begin{array}{r}\text { Ausbildung in einem anderen Beruf } \\
\text { Laufbahnperspektiven }\end{array}$ & 13 & 18 & 14 & 11 & 1 \\
\hline Laufbahnperspektiven & 12 & 12 & 4 & 36 & 2 \\
\hline erkennung und Wertschätzung bei der Arbeit & 12 & 11 & 8 & 26 & 5 \\
\hline Berufliche Laufbahn Partnerln & (1) & 10 & 20 & 5 & 8 \\
\hline Arbeitsbedingungen & 6 & 8 & 4 & 5 & 6 \\
\hline Persönliche Umstände & 6 & 6 & 8 & 5 & 8 \\
\hline Verdienst und Verdienstaussichten & 6 & 5 & 3 & 13 & 3 \\
\hline Anderes & 5 & 8 & 3 & 6 & 1 \\
\hline \multirow{2}{*}{$\begin{array}{r}\text { Familienplanung, erschwerter Wiedereinstieg } \\
\text { Berufliche Entwicklung, fehlende } \\
\text { Weiterbildungsmöglichkeiten }\end{array}$} & o & 6 & 4 & 3 & 4 \\
\hline & 2 & 4 & & 2 & \\
\hline Arbeitslosigkeit, Stellenverlust & $\$$ & & 3 & & 4 \\
\hline keine Angabe & 6 & 8 & 6 & 3 & 2 \\
\hline
\end{tabular}

Quelle: gfs.bern, Laufbahnbefragung von Schweizer ÄrztInnen 2016. N = 420: Befragte, die die kurative Tätigkeit vor Erreichen des Pensionsalters aufgegeben haben. Frage 21: „Welches sind die wichtigsten Gründe dafür, dass Sie aktuell nicht kurativ tätig sind? Bitte kreuzen Sie maximal drei Gründe an." Angaben in Prozent der jeweiligen Spalte (Alle, Typ 1 bis Typ 4)

Abbildung 2: Gründe für die Aufgabe der kurativen Tätigkeit.

79 Prozent geben das Erreichen des Facharzttitels als wahrscheinlich oder eher wahrscheinlich an.

\section{Gründe für den Ausstieg}

Die nicht mehr kurativ tätigen Ärzte geben für ihren Ausstieg vorzugsweise Gründe an, die eng mit der ärztlichen Arbeitssituation zusammenhängen (Abb. 2). Das Arbeitspensum und die Arbeitszeiten werden mit Abstand am häufigsten genannt: Gut ein Drittel der Befragten (34\%) bezeichnet diesen Punkt als einen der drei wichtigsten Gründe für die Aufgabe der kurativen Tätigkeit. Die Vereinbarkeit der ärztlichen Tätigkeit mit der Kinderbetreuung (22\%) und die Arbeitsinhalte selbst (21\%) wurden von gut jeder fünften ausgestiegenen Person vorgebracht. Auch der nächste Faktor, das Anforderungsniveau mit 16 Prozent, bezieht sich auf die Arbeit selbst. Erst danach folgen anders gelagerte Gründe wie die Gesundheit, die Neuorientierung oder der Ruhestand (es sei daran erinnert, dass in der Auswertung nur Personen berücksichtigt sind, die vor dem Erreichen des 65. Altersjahrs aus der kurativen Tätigkeit ausgestiegen sind).
Hinsichtlich der Häufigkeit der verschiedenen Gründe gleicht Typ 1 (frühe Berufswechselnde) am stärksten der Gesamtheit aller aus der kurativen Tätigkeit ausgestiegenen Personen. Von den frühen Berufswechselnden bezeichnen sogar 43 Prozent Pensum und Arbeitszeiten als wichtigen Ausstiegsgrund. Überdurchschnittlich vertreten sind mit gut einem Viertel (27\%) der Nennungen auch die mangelnde Vereinbarkeit des Arztberufs mit der Kinderbetreuung sowie die Arbeitsinhalte. Schliesslich spielt die Neuorientierung mit 22 Prozent, mehr als bei jedem anderen Typ, ebenfalls eine Rolle. Ausgestiegene von Typ 2 (frühe Berufsaussteigende) bringen das Pensum und die Arbeitszeiten (34\%) fast ebenso häufig wie die mangelnde Vereinbarkeit mit der Kinderbetreuung (32\%) als Grund für die Aufgabe der kurativen Tätigkeit vor. Gesundheitliche Gründe sind bei diesem Typ mit fast ein Viertel der Angaben (23\%) der dritthäufigste Faktor. Jede fünfte Person in diesem Typ nennt ausserdem die Laufbahn ihres Partners, ihrer Partnerin als Ausstiegsgrund.

Auch bei Typ 3 (späte Berufswechselnde) gab mehr als ein Drittel der Befragten (37\%) das Pensum und die Arbeitszeiten als einen der drei wichtigsten Ausstiegsgründe an. Für praktisch gleich viele Befragte (36\%) waren die Laufbahnperspektiven ein wichtiger Faktor, so viel wie in keinem anderen Typ. Ebenfalls sehr bedeutend sind die Arbeitsinhalte mit 34 Prozent der Nennungen.

Bei Typ 4 (späte Berufsaussteigende) sind schliesslich zwei Ausstiegsgründe dominant. Die Mehrheit dieses Typs (59\%) geht von der kurativen Tätigkeit direkt in den Ruhestand. Fast ein Drittel (31\%) bringt gesundheitliche Gründe für die Aufgabe der kurativen Tätigkeit vor.

Bei Frauen geben häufig andere Gründe den Ausschlag für die Aufgabe der kurativen Tätigkeit. Sie nennen die mangelnde Vereinbarkeit des Arztberufes mit der Kinderbetreuung (41\%), während von den Männern nur 6 Prozent diesen Grund aufführen.

Die nicht mehr kurativ tätigen Ärzte wurden zuletzt nach ihrem Erleben der ärztlichen Berufstätigkeit befragt. Für 77 Prozent der Befragten $(\mathrm{N}=314)$ war die Arbeitszeit zu lang und für 68 Prozent waren die Einsatzzeiten zu unregelmässig. Die frühen Berufswechselnden (Typ 1) sind am seltensten zufrieden mit der ärztlichen Berufstätigkeit, die späteren Berufsaussteigenden (Typ 4) am häufigsten. Ein ähnliches Bild zeigt sich bei der Einschätzung zur Vereinbarkeit von Beruf mit Familie, Freundeskreis und Freizeit (Abb. 3). Je früher sich der Arzt für einen Ausstieg entschieden hat, desto weniger war die Vereinbarkeit mit der Kinderbetreuung gegeben - bloss 3 Prozent der frühen Berufswechselnden erachteten dies so. Obwohl in der Frage 
Vergleich nach Typen und Geschlecht:

Anteil Kategorien «Vereinbarkeit war eindeutig gegeben» + «Vereinbarkeit war eher gegeben» Es werden nur signifikante Gruppenunterschiede ausgewiesen

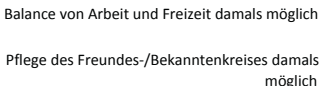

möglich

Laufbahn mit Partnerin damals vereinbar

Betreuung der Kinder damals vereinbar

\begin{tabular}{|c|c|c|c|c|c|c|}
\multicolumn{1}{c}{ Alle } & \multicolumn{1}{c}{ Tyр 1 } & \multicolumn{1}{c}{ Typ 2 } & \multicolumn{1}{c}{ Tyр 3 } & \multicolumn{1}{c}{ Typ 4 } & Männer & \multicolumn{1}{c|}{ Frauen } \\
\hline 37 & 26 & 30 & 44 & 48 & 43 & 32 \\
\hline 41 & 33 & 40 & 50 & 45 & 48 & 35 \\
\hline 40 & 35 & 40 & 45 & 43 & 45 & 35 \\
\hline 14 & 3 & 8 & 14 & 30 & & \\
\hline
\end{tabular}

Quelle: gfs.bern, Laufbahnbefragung von Schweizer ÄrztInnen 2016. N = 314: Befragte, die die kurative Tätig keit vor Erreichen des Pensionsalters aufgegeben haben (ohne Befragte, die die Weiterbildung nicht angetreten haben). Frage 20: «Wenn Sie an Ihre letzte kurative Tätigkeit zurückdenken: Zu welchem Grad war Ihre Arbeit mit Ihren Ansprüchen an Freizeit, Partnerschaft und Familie vereinbar?» Angaben in Prozent der jeweiligen Kategorie (Alle, Typ).

Abbildung 3: Einschätzung zur Vereinbarkeit.

der Vereinbarkeit kein signifikanter Unterschied zwischen den Geschlechtern feststellbar ist, bewegt diese Einschätzung die Frauen offenbar viel mehr zum effektiven Ausstieg aus der kurativen Tätigkeit.

\section{Fazit}

Die Analyse zeigt, dass der geschätzte Anteil der Ärztinnen und Ärzte, welche zwischen 1980 und 2009 ihr Ärztediplom in der Schweiz erworben haben und nicht mehr kurativ am Patienten tätig sind, zwischen 8,4 und 12,9 Prozent liegt. Der Frauenanteil ist etwas höher als derjenige der Männer. Für den grössten Teil der Ärzte ist der Ausstieg definitiv - nur gerade jeder Zehnte geht davon aus, eher oder sehr wahrscheinlich wieder in den Arztberuf zurückzukehren. Hierbei gelingt es den ausgestiegenen Männern besser als den Frauen, ihre ärztlichen Qualifikationen zu verwerten.

Korrespondenz:

VSAO

Bahnhofplatz 10A

CH-3011 Bern

sekretariat[at]vsao.ch

FMH

Elfenstrasse 18

Postfach 300

3000 Bern 15

ddq[at]fmh.ch
Die Ärzte, welche ihre kurative Tätigkeit vor Erreichen des Pensionsalters aufgegeben haben, lassen sich in drei etwa gleich grosse Gruppen teilen. Ein erstes Drittel hat die Weiterbildung gar nicht angetreten, das zweite Drittel hat die kurative Tätigkeit in der Assistenzzeit aufgegeben und das dritte Drittel ist erst nach dem Erwerb des Facharzttitels ausgestiegen. Die frühen Berufswechselnden oder die frühen Berufsaussteigen- den sind im Durchschnitt zwischen 30 und 35 Jahre alt, die späten Berufswechselnden etwas mehr als 40 Jahre und die späten Berufsaussteigenden durchschnittlich 52 Jahre alt.

Folgende Faktoren können mitentscheidend für den Ausstieg aus der kurativen Tätigkeit sein:

- Rund ein Drittel nennt als wichtigsten Grund das Arbeitspensum und die Arbeitszeiten.

- Für je gut ein Fünftel der Befragten waren die mangelnde Vereinbarkeit des Arztberufes mit der Kinderbetreuung oder die Arbeitsinhalte ausschlaggebend.

- Die ausscheidenden Ärztinnen und Ärzte haben ein kritischeres Bild des Arztberufes als ihre noch kurativ tätigen Kollegen.

- Die nicht mehr kurativ tätigen Ärzte machen die negativen Erfahrungen wissentlich, d.h. sie kennen die Herausforderungen bereits am Ende ihres Medizinstudiums.

- Eine unbefriedigende Situation im Arztberuf wird etwa gleich häufig als Grund für die Aufgabe angegeben wie attraktive Alternativen, die sich eröffnen.

\section{Schlussfolgerung}

Pro Abschlussjahrgang Humanmedizin verliert die Schweiz rund 80 Ärztinnen und Ärzte, die nicht mehr kurativ am Patienten tätig sind. Auch wenn dieser Anteil relativ tief ist, so ist doch jeder Ausstieg einer zu viel. Besonders bedenklich ist, dass sich nur 9 Prozent der Aussteiger überhaupt eine Rückkehr in die kurative Tätigkeit vorstellen können. Anders gesagt: Wer einmal aus der kurativen Tätigkeit aussteigt, wird kaum mehr zurückkehren. Umso dringlicher sind deshalb Massnahmen, die dafür sorgen, dass Ärztinnen und Ärzte ihre Tätigkeit am Patienten weiterverfolgen:

- Die Arbeitszeiten und die Einsatzzeiten attraktiver gestalten.

- Die Vereinbarkeit von Beruf und Familie/Kinderbetreuung besser gewährleisten und sichtbarer machen.

- Die Attraktivität und die Arbeitsinhalte verbessern. 